\title{
A note on the ternary Diophantine equation
}

$$
x^{2}-y^{2 m}=z^{n}
$$

\section{Attila Bérczes, Maohua Le, István Pink, and Gökhan Soydan}

\begin{abstract}
Let $\mathbb{N}$ be the set of all positive integers. In this paper, using some known results on various types of Diophantine equations, we solve a couple of special cases of the ternary equation $x^{2}-y^{2 m}=z^{n}, x, y, z, m, n \in$ $\mathbb{N}, \operatorname{gcd}(x, y)=1, m \geq 2, n \geq 3$.
\end{abstract}

\section{Introduction}

Let $\mathbb{Z}, \mathbb{N}$ be the sets of all integers and positive integers, respectively. In 1965 , the first work on the title equation was done by C. Ko [9]. He showed that $x^{2}-y^{n}=1$ has no solution with $y>2$ and $n \geq 2$. In 1997, Y. Bugeaud [4] proved that Diophantine equations

$$
x^{2}-2^{m}= \pm y^{n}, \quad x, y, m, n \in \mathbb{N}, \operatorname{gcd}(x, y)=1, y>1, n>2
$$

have only finitely many solutions where $n \leq 5.5 \cdot 10^{5}$. In the same year, same author gave some partial results to prove the equation

$$
x^{2}-p^{m}= \pm y^{n}, \quad x, y, m, n \in \mathbb{N}, \operatorname{gcd}(x, y)=1, n \geq 3
$$

where $p$ is odd prime [5]. In 2003, S. Siksek [14] gave all the solutions of the Diophantine equation

$$
x^{2}-2^{k} \cdot z^{p}=y^{p}, \quad p \geq 7, k \geq 2
$$

Key Words: higher Diophantine equation,exponential Diophantine equation, generalized Fermat equation, ternary equation

2010 Mathematics Subject Classification: 11D41,1D61

Received: 20.11 .2020

Accepted: 30.12 .2020 
in pairwise coprime integers $x, y, z$. And in the same year, W. Ivorra [8] studied the equations

$$
x^{p}+2^{\beta} y^{p}=z^{2}
$$

and

$$
x^{p}+2^{\beta} y^{p}=2 z^{2},
$$

where $p \geq 7,0 \leq \beta \leq p-1$. And he also solved

$$
x^{2}-2^{m}=y^{n}, m>1 \text {. }
$$

Up to day the famous equation $x^{2}-2=y^{n}$ is still unsolved and is one of the most exciting questions on "classical Diophantine equations".

Another approach to the title equation is to consider it as a generalized Fermat equation. Fix nonzero integers $A, B$ and $C$. For given positive integers $p, q, r$ satisfying $1 / p+1 / q+1 / r<1$, the generalized Fermat equation

$$
A x^{p}+B y^{q}=C z^{r}
$$

has only finitely many primitive integer solutions. Modern techniques coming from Galois representations and modular forms (methods of Frey-Hellegouarch curves and variants of Ribet's lever-lowering theorem, and of course, the modularity of elliptic curves or abelian varieties over the rationals or totally real number fields) allow to give partial (sometimes complete) results concerning the set of solutions to (1.1) (usually, when a radical of $A B C$ is small), at least when $(p, q, r)$ is of the type $(n, n, n),(n, n, 2),(n, n, 3),(2 n, 2 n, 5),(2,4, n)$, $(2,6, n),(2, n, 4),(2, n, 6),(3,3, p),(2,2 n, 3),(2,2 n, 5)$. Recently,two survey papers concerning solving the equation (1.1) when $A B C=1$ were published by M. Bennett, I. Chen, S. Dahmen and S. Yazdani [1] and by M. Bennett, P. Mihăilescu and S. Siksek [2].

In this paper, using some known results on various types of Diophantine equations, we obtain the following results of the equation

$$
x^{2}-y^{2 m}=z^{n}, x, y, z, m, n \in \mathbb{N}, \operatorname{gcd}(x, y)=1, m \geq 2, n \geq 3 .
$$

Theorem 1.1. (1.2) has only the solution

$$
(x, y, z, m, n)=(122,11,3,2,5)
$$

satisfying $2 \mid m$ and $n$ is an odd prime with

$$
n> \begin{cases}3, & \text { if } 2 \nmid z, \\ 5, & \text { if } 2 \mid z .\end{cases}
$$


Theorem 1.2. (1.2) has no solutions $(x, y, z, m, n)$ satisfying $y$ is an odd prime and $2 \mid n$.

Theorem 1.3. For any fixed odd positive integer $n$ with $n>3$, (1.2) has only finitely many solutions $(x, y, z, m, n)$ satisfying $2 \mid x, y$ is an odd prime and $2 \nmid m$.

For any positive integer $r$, let

$$
a_{r}=\frac{1}{2}\left(\alpha^{r}+\bar{\alpha}^{r}\right), b_{r}=\frac{1}{2 \sqrt{3}}\left(\alpha^{r}-\bar{\alpha}^{r}\right),
$$

where $\alpha=7+\sqrt{3}$ and $7-4 \sqrt{3}$.

Theorem 1.4. If $(x, y, z, m, n)$ is a solution of (1.2) satisfying $2 \mid x, y$ is an odd prime and $n=3$, then

$$
(x, y, z, m, n)=\left(b_{2^{s}}^{3}+3 b_{2^{s}}, a_{2^{s}}, b_{2^{s}}^{2}-1,2,3\right)
$$

$s \in \mathbb{Z}, s \geq 0$.

Remark 1. By Theorem 1.4, we can obtain the solutions $(x, y, z, m, n)=$ $(76,7,15,2,3),(175784,97,3135,2,3)$ and so on.

Theorem 1.5. (1.2) has only the solution (1.3) satisfying $2 \mid x, y$ is an odd prime, $m<7$ and $n=5$.

\section{Preliminaries}

Let $D$ be a nonsquare positive integer, and let $h(4 D)$ denote the class number of binary quadratic primitive forms with discriminant $4 D$.

Lemma 2.1. ([12]) The equation

$$
u^{2}-D v^{2}=1, u, v \in \mathbb{Z}
$$

has positive integer solution $(u, v)$, and it has a unique positive integer solution $\left(u_{1}, v_{1}\right)$ such that $u_{1}+v_{1} \sqrt{D}<u+v \sqrt{D}$, where $(u, v)$ through all positive integer solutions of (2.1). The solution $\left(u_{1}, v_{1}\right)$ is called the least solution of (2.1). Every solution $(u, v)$ of (2.1) can be expressed as

$$
u+v \sqrt{D}=\lambda_{1}\left(u_{1}+\lambda_{2} v_{1} \sqrt{D}\right)^{s}, s \in \mathbb{Z}, s \geq 0, \lambda_{1}, \lambda_{2} \in\{1,-1\} .
$$

Lemma 2.2. Let $a_{r}, b_{r}(r=1,2, \cdots)$ de defined as in (1.5). Then, $(u, v)=$ $\left(a_{r}, b_{r}\right)(r=1,2, \cdots)$ are all positive integer solutions of the equation

$$
u^{2}-3 v^{2}=1, u, v \in \mathbb{Z}, 2 \mid v .
$$


Proof. Notice that $(u, v)$ is a solution of $(2.2)$ if and only if $\left(u^{\prime}, v^{\prime}\right)=(u, v / 2)$ is a solution of the equation

$$
u^{\prime 2}-12 v^{\prime 2}=1, u^{\prime}, v^{\prime} \in \mathbb{Z}
$$

and $\left(u^{\prime}, v^{\prime}\right)=(7,2)$ is the least solution of (2.3). Therefore, by Lemma 2.1, we obtain the lemma immediately.

Lemma 2.3. ([12]) If the equation

$$
U^{2}-D V^{2}=4, U, V \in \mathbb{Z}, 2 \nmid U V
$$

has solutions $(U, V)$, then it has a unique positive integer solution $\left(U_{1}, V_{1}\right)$ such that $U_{1}+V_{1} \sqrt{D} \leq U+V \sqrt{D}$, where $(U, V)$ through all positive integer solutions of (2.4). The solution $\left(U_{1}, V_{1}\right)$ is called the least solution of (2.4). Then, the least solution $\left(u_{1}, v_{1}\right)$ of $(2.1)$ satisfies

$$
u_{1}+v_{1} \sqrt{D}=\left(\frac{U_{1}+V_{1} \sqrt{D}}{2}\right)^{3}
$$

Lemma 2.4. Let $k$ be an integer such that $|k|>1$ and $\operatorname{gcd}(D, k)=1$. If $2 \nmid k$ and the equation

$$
X^{2}-D Y^{2}=k^{Z}, X, Y, Z \in \mathbf{Z}, \operatorname{gcd}(X, Y)=1, Z>0
$$

has solutions $(X, Y, Z)$, then every solution $(X, Y, Z)$ of (2.6) can be expressed as

$$
\begin{aligned}
& Z=h t, t \in \mathbf{N}, \\
& X+Y \sqrt{D}=(f+\lambda g \sqrt{D})^{t}(u+v \sqrt{D}), \lambda \in\{1,-1\},
\end{aligned}
$$

where $(u, v)$ is a solution of (2.1), $f, g$ and $h$ are positive integers satisfying

$$
\begin{gathered}
f^{2}-D g^{2}=k^{h}, \operatorname{gcd}(f, g)=1, \\
1<\left|\frac{f+g \sqrt{D}}{f-g \sqrt{D}}\right|<u_{1}+v_{1} \sqrt{D}, \\
h(4 D) \equiv 0 \quad(\bmod h)
\end{gathered}
$$

where $\left(u_{1}, v_{1}\right)$ is the least solution of (2.1).

Proof. This lemma is special case of results of [10] and [17] for $D_{1}=1, D_{2}>0$ and $2 \nmid k$. 
Lemma 2.5. If $3 \nmid h(4 D),(2.4)$ has solutions $(U, V)$ and the equation

$$
X^{\prime 2}-D Y^{\prime 2}=Z^{\prime 3}, X^{\prime}, Y^{\prime}, Z^{\prime} \in \mathbb{Z}, \operatorname{gcd}\left(X^{\prime}, Y^{\prime}\right)=1,\left|Z^{\prime}\right|>1
$$

has solutions $\left(X^{\prime}, Y^{\prime}, Z^{\prime}\right)$, then every solution $\left(X^{\prime}, Y^{\prime}, Z^{\prime}\right)$ of $(2.11)$ can be expressed as

$$
\begin{gathered}
X^{\prime}+Y^{\prime} \sqrt{D}=\lambda_{1}\left((f+\lambda g \sqrt{D})\left(\frac{U_{1}+\lambda_{2} V_{1} \sqrt{D}}{2}\right)^{s}\right)^{3}, \\
s \in \mathbb{Z}, s \geq 0, \lambda, \lambda_{1}, \lambda_{2} \in\{1,-1\}, \\
Z^{\prime}=f^{2}-D g^{2}, f, g \in \mathbb{N}, \operatorname{gcd}(f, g)=1,
\end{gathered}
$$

where $\left(U_{1}, V_{1}\right)$ is the least solution of (2.4), $f$ and $g$ satisfy (2.9)

Proof. We now assume that $\left(X^{\prime}, Y^{\prime}, Z^{\prime}\right)$ is a solution of (2.11). Since $\operatorname{gcd}\left(X^{\prime}, D Y^{\prime}\right)=1$, by $(2.11)$, we have $\operatorname{gcd}\left(D, z^{\prime}\right)=1$. On the other hand, since (2.4) has solutions $(U, V)$ with $2 \nmid U V$, we have $D \equiv D V^{2} \equiv U^{2}-4 \equiv$ $1-4 \equiv 5(\bmod 8)$. Hence, if $2 \mid Z^{\prime}$, then from $(2.11)$ we get $2 \nmid X^{\prime} Y^{\prime}$ and $0 \equiv Z^{\prime 3} \equiv X^{\prime 2}-D Y^{\prime 2} \equiv 1-D \equiv 4 \not \equiv 0(\bmod 8)$, a contradiction. So we have $2 \nmid Z^{\prime}$.

Since $\operatorname{gcd}\left(D, Z^{\prime}\right)=1,2 \nmid Z^{\prime}$ and $(2.6)$ has a solution $(X, Y, Z)=\left(X^{\prime}, Y^{\prime}, 3\right)$ for $k=Z^{\prime}$, by Lemma 2.4, we have

$$
\begin{gathered}
3=h t, t \in \mathbb{N}, \\
X^{\prime}+Y^{\prime} \sqrt{D}=(f+\lambda g \sqrt{D})^{t}(u+v \sqrt{D}), \lambda \in\{1,-1\},
\end{gathered}
$$

where $(u, v)$ is a solution of (2.1), $f, g$ and $h$ are positive integers satisfying (2.8), (2.9) and (2.10). Further, since $3 \nmid h(4 D)$, by (2.10) and (2.14), we get $h=1$ and $t=3$. Hence, by (2.8) and (2.15), we obtain (2.13) and

$$
X^{\prime}+Y^{\prime} \sqrt{D}=(f+\lambda g \sqrt{D})^{3}(u+v \sqrt{D}), \lambda \in\{1,-1\}
$$

respectively. Furthermore, since (2.4) has solutions $(U, V)$, by Lemmas 2.1 and 2.3 , we have

$$
u+v \sqrt{D}=\lambda_{1}\left(\frac{U_{1}+\lambda_{2} V_{1} \sqrt{D}}{2}\right)^{3 s}, s \in \mathbb{Z}, s \geq 0, \lambda_{1}, \lambda_{2} \in\{1,-1\},
$$

where $\left(U_{1}, V_{1}\right)$ is the least solution of (2.4). Therefore, substitute (2.17) into (2.16), we get (2.12). Thus, the lemma is proved. 
Lemma 2.6. The equation

$$
4-5 A^{2}=-B^{3}, A, B \in \mathbb{N}, \operatorname{gcd}(A, B)=1
$$

has only the solution $(A, B)=(1,1)$

Proof. Obviously, (2.18) has only the solution $(A, B)=(1,1)$ with $B=1$. We now assume that $(A, B)$ is a solution of (2.18) with $B>1$. Then, (2.11) has a solution

$$
\left(X^{\prime}, Y^{\prime}, Z^{\prime}\right)=(2, A,-B) \text { for } D=5 .
$$

Notice that $h(20)=1$, the least solution of $(2.1)$ is $\left(u_{1}, v_{1}\right)=(9,4)$ for $D=5$, (2.4) has solutions $(U, V)$ for $D=5$ and its least solution is $\left(U_{1}, V_{1}\right)=(3,1)$. Applying Lemma 2.5 to (2.19), we get

$$
2+A \sqrt{5}=\lambda_{1}\left((f+\lambda g \sqrt{5})\left(\frac{3+\lambda_{2} \sqrt{5}}{2}\right)^{s}\right)^{3}, s \in \mathbb{Z}, s \geq 0, \lambda, \lambda_{1}, \lambda_{2} \in\{1,-1\}
$$

and

$$
-B=f^{2}-5 g^{2}, f, g \in \mathbb{N}, \operatorname{gcd}(f, g)=1 .
$$

Let

$$
\begin{aligned}
& \beta=\frac{F+G \sqrt{5}}{2}=(f+\lambda g \sqrt{5})\left(\frac{3+\lambda_{2} \sqrt{5}}{2}\right)^{s}, \\
& \bar{\beta}=\frac{F-G \sqrt{5}}{2}=(f-\lambda g \sqrt{5})\left(\frac{3-\lambda_{2} \sqrt{5}}{2}\right)^{s} .
\end{aligned}
$$

By Lemmas 2.1 and 2.3, we have

$$
\left(\frac{3+\lambda_{2} \sqrt{5}}{2}\right)^{s}=\left\{\begin{array}{lll}
u+v \sqrt{5}, & \text { if } s \equiv 0 \quad(\bmod 3), \\
(u+v \sqrt{5})\left(\frac{3+\lambda_{2} \sqrt{5}}{2}\right), & \text { if } s \equiv 1 \quad(\bmod 3), \\
(u+v \sqrt{5})\left(\frac{7+3 \lambda_{2} \sqrt{5}}{2}\right), & \text { if } s \equiv 2 \quad(\bmod 3),
\end{array}\right.
$$

where $(u, v)$ is a solution of $(2.1)$ for $D=5$. Hence, by (2.21), (2.22) and (2.23), we get

$$
F^{2}-5 G^{2}=-4 B, F, G \in \mathbb{Z} .
$$

Substitute (2.22) into (2.20), we have

$$
2+A \sqrt{5}=\lambda_{1} \beta^{3}, \quad 2-A \sqrt{5}=\lambda_{1} \bar{\beta}^{3} .
$$


Eliminating $A$ from (2.25), we get

$$
4=\lambda_{1}\left(\beta^{3}+\bar{\beta}^{3}\right) .
$$

Further, by (2.22) and (2.24), we have

$$
\beta+\bar{\beta}=F, \quad \beta \bar{\beta}=-B .
$$

Therefore, by (2.26) and (2.27), we get

$$
\begin{aligned}
& 4=\lambda_{1}\left(\beta^{3}+\bar{\beta}^{3}\right)=\lambda_{1}(\beta+\bar{\beta})\left((\beta+\bar{\beta})^{2}-3 \beta \bar{\beta}\right) \\
& =\lambda_{1} F\left(F^{2}+3 B\right)=\lambda_{1} F\left(F^{2}-\frac{3}{4}\left(F^{2}-5 G^{2}\right)\right) \\
& =\frac{\lambda_{1} F}{4}\left(F^{2}+15 G^{2}\right),
\end{aligned}
$$

whence we obtain

$$
16=|F|\left(F^{2}+15 G^{2}\right) .
$$

Since $F$ and $G$ are integers, by (2.28), we can only get

$$
(F, G)=( \pm 1, \pm 1) \text {. }
$$

But, by (2.24) and (2.29), we deduce that $B=1$, a contradiction. It implies that (2.18) has no solutions $(A, B)$ with $B>1$. Thus, the lemma is proved.

Lemma 2.7. ([12]) Every solution $(X, Y, Z)$ of the equation

$$
X^{2}+Y^{2}=Z^{2}, X, Y, Z \in \mathbb{N}, \operatorname{gcd}(X, Y)=1,2 \mid Y
$$

can be expressed as

$$
\begin{aligned}
& X=f^{2}-g^{2}, Y=2 f g, Z=f^{2}+g^{2} f, g \in \mathbb{N} \\
& f>g, \operatorname{gcd}(f, g)=1, f \not \equiv g \quad(\bmod 2) .
\end{aligned}
$$

Lemma 2.8. ([6]) The equation

$$
1+3 X^{2}=Y^{r}, X, Y, r \in \mathbb{N}, r \geq 3
$$

has no solutions $(X, Y, r)$.

Lemma 2.9. ([13]) Let $0 \neq b \in \mathbb{Z}$ and let $f(X) \in \mathbb{Q}[X]$ be a polynomial with at least two distinct zeroes. Then the equation

$$
f(X)=b Y^{n}, n>2
$$

in integers $X, Y>1, n$ implies that $n<C$ where $C=(f, b)$ is an effectively computable constant. 
Lemma 2.10. ([11]) The equation

$$
X^{r}-Y^{s}=1, X, Y, r, s \in \mathbb{N}, \min \{X, Y, r, s\}>1
$$

has only the solution $(X, Y, r, s)=(3,2,2,3)$.

Lemma 2.11. ([7],[15],[16]) The equation

$X^{r} \pm Y^{r} \pm 2^{\delta} Z^{r}=0, X, Y, Z, r, \delta \in \mathbb{Z}, \operatorname{gcd}(X, Y)=1,|X Y Z|>1, r \geq 3, \delta \geq 0$ has no solutions $(X, Y, Z, r, \delta)$.

Lemma 2.12. (Theorem 1.1 of [3]) The equation

$$
2 X^{2}=Y^{r}+Z^{r}, X, Y, Z, r \in \mathbb{Z}, X Y Z \neq 0, Y>Z, \operatorname{gcd}(Y, Z)=1, r \geq 4
$$

has only the solutions $(X, Y, Z, r)=( \pm 11,3,-1,5)$.

Lemma 2.13. (Theorem 1.2 of [3]) The equation

$$
\begin{aligned}
& X^{2}=Y^{r}+2^{\delta} Z^{r}, X, Y, Z, r, \delta \in \mathbb{Z}, \operatorname{gcd}(X, Y)=1, \\
& |Y Z|>1, r \text { is an odd prime with } r \geq 7, \delta \geq 2
\end{aligned}
$$

has no solutions $(X, Y, Z, r, \delta)$.

\section{Proof of Theorem 1.1}

We now assume that $(x, y, z, m, n)$ is a solution of (1.2) satisfying $2 \mid m$ and $n$ is an odd prime with (1.4). If $2 \nmid z$, then we have

$$
x+y^{m}=a^{n}, x-y^{m}=b^{n}, z=a b, a, b \in \mathbb{N}, a>b, \operatorname{gcd}(a, b)=1,2 \nmid a b .
$$

Eliminating $x$ from (3.1), we get

$$
2 y^{m}=a^{n}-b^{n} .
$$

Since $2 \mid m$ and $n$ is an odd prime with $n>3$, we see from (3.2) that (2.32) has a solution $(X, Y, Z, r)=\left(y^{m / 2}, a,-b, n\right)$. Therefore, by Lemma 2.12 , we get only the solution (1.3) by (3.1).

If $2 \mid z$, then from (1.2) we get

$x+y^{m}=\left\{\begin{array}{l}2 a^{n}, \\ 2^{n-1} b^{n},\end{array} \quad x-y^{m}=\left\{\begin{array}{l}2^{n-p}, \\ 2 a^{n},\end{array} \quad z=2 a b, a, b \in \mathbb{N}, \operatorname{gcd}(a, b)=1,2 \nmid a\right.\right.$. 
By (3.3), we hve

$$
y^{m}=\left\{\begin{array}{l}
a^{n}-2^{n-2} b^{n}, \\
2^{n-2} b^{n}-a^{n} .
\end{array}\right.
$$

But, since $2 \mid m$ and $n$ is an odd prime with $n>5$, by Lemma 2.13, (3.4) is false. Thus, the theorem is proved.

\section{Proof of Theorem 1.2}

We now assume that $(x, y, z, m, n)$ is a solution of (1.2) satisfying $y$ is an odd prime and $2 \mid n$. By (1.2), then (2.30) has a solution $(X, Y, Z)=\left(y^{m}, z^{n / 2}, x\right)$. Hence, by Lemma 2.7, we have

$$
\begin{aligned}
& y^{m}=f^{2}-g^{2}, z^{n / 2}=2 f g, x=f^{2}+g^{2} f, g \in \mathbb{N}, \\
& f>g, \operatorname{gcd}(f, g)=1, f \not \equiv g \quad(\bmod 2) .
\end{aligned}
$$

Since $y$ is an odd prime, we see from the first equality of (4.1) that

$$
f-g=1
$$

and

$$
y^{m}=f+g=2 f-1=2 g+1 .
$$

On the other hand, by the second equality of (4.1), we have

$$
\begin{aligned}
& z=2 a b, a, b \in \mathbb{N} \operatorname{gcd}(a, b)=1,2 \nmid a, \\
& f=\left\{\begin{array}{ll}
a^{n / 2}, & \text { if } 2 \nmid f \\
2^{n / 2-1} b^{n / 2},
\end{array} \quad g= \begin{cases}2^{n / 2-1} b^{n / 2}, & \text { if } 2 \mid f . \\
a^{n / 2}, & \end{cases} \right.
\end{aligned}
$$

Substitute (4.4) into (4.2), we get

$$
a^{n / 2}-2^{n / 2-1} b^{n / 2}= \pm 1
$$

By Lemma 2.11, if $n / 2 \geq 3$, then (4.5) is false. So we have $n / 2=2$, since $n \geq 3$.

Since $n / 2=2$, by $(4.5)$, we get

$$
a^{2}-2 b^{2}= \pm 1
$$

Hence, by $(4.3),(4.4)$ and $(4.6)$, we have

$$
y^{m}=4 b^{2} \pm 1 \text {. }
$$

But, since $m \geq 2$, by Lemma 2.10, (4.7) is false. Thus, the theorem is proved. 


\section{$5 \quad$ Proof of Theorem 1.3}

We now assume that $(x, y, z, m, n)$ is a solution of (1.2) satisfying $2 \mid x, y$ is an odd prime and $2 \nmid m$. Since $2 \mid x$, by (1.2), we have $2 \nmid z$. Hence, by the proof of Theorem 1.1, we get (3.1) and (3.2). Further, since $y$ is an odd prime, by (3.2), we have

$$
a-b=2
$$

and

$$
\frac{a^{n}-b^{n}}{a-b}=y^{m} .
$$

For any positive integer $n$ with $n \geq 3$, let

$$
f_{n}(X)=\frac{1}{2}\left((X+2)^{n}-X^{n}\right)=\sum_{i=1}^{n}\left(\begin{array}{c}
n \\
i
\end{array}\right) 2^{i-1} X^{n-i} .
$$

Then $f_{n}(X) \in \mathbb{Z}[X]$ is a polynomial of degree $n-1$. Further, let $\zeta_{j}(j=$ $0,1, \cdots, n-1)$ denote all $n$-th unit roots with $\zeta_{0}=1$. Obviously, by (5.3), $2 /\left(\zeta_{j}-1\right)(j=1, \cdots, n-1)$ are total roots of $f_{n}(X)$. By $(5.1),(5.2)$ and (5.3), we have

$$
f_{n}(b)=y^{m} .
$$

Since $m \geq 3$, applying Lemma 2.9 to (5.4), we can obtain the theorem immediately.

\section{Proof of Theorem 1.4}

We now assume that $(x, y, z, m, n)$ is a solution of (1.2) satisfying $2 \mid x, y$ is an odd prime and $n=3$. Then, since $2 \nmid z$, by (3.1) and (3.2), we get (5.1) and

$$
\frac{a^{3}-b^{3}}{a-b}=y^{m}
$$

Substitute (5.1) into (6.1), we have

$$
1+3(b+1)^{2}=y^{m} .
$$

Hence, applying Lemma 2.8 to $(6.2)$, we get $m<3$, So we have $m=2$, since $m \geq 2$.

Since $m=2$ and $2 \nmid b$ by (3.1), we see from (6.2) that (2.2) has a positive integer solution $(u, v)=(y, b+1)$. Hence, by Lemma 2.2 , we have

$$
y=a_{r}, \quad b+1=b_{r}, r \in \mathbb{N} .
$$


If $r$ has an odd divisor $d$ with $d>1$, then from (1.5) and (6.3) we get

$$
\begin{aligned}
& y=a_{r}=\frac{\alpha^{r}+\bar{\alpha}^{r}}{2}=\frac{\left(\alpha^{r / d}\right)^{d}+\left(\bar{\alpha}^{r / d}\right)^{d}}{2} \\
& =\left(\frac{\alpha^{r / d}+\bar{\alpha}^{r / d}}{2}\right)\left(\frac{\alpha^{r}+\bar{\alpha}^{r}}{\alpha^{r / d}+\bar{\alpha}^{r / d}}\right)=a_{r / d}\left(\frac{\alpha^{r}+\bar{\alpha}^{r}}{\alpha^{r / d}+\bar{\alpha}^{r / d}}\right),
\end{aligned}
$$

where $a_{r / d}$ and $\left(\alpha^{r}+\bar{\alpha}^{r}\right) /\left(\alpha^{r / d}+\bar{\alpha}^{r / d}\right)$ are positive integers greater than 1 . But, since $y$ is an odd prime, (6.4) is false. It implies that

$$
r=2^{s}, s \in \mathbb{Z}, s \geq 0 .
$$

Thus, by (3.1), (6.3) and (6.5), we get (1.6). The theorem is proved.

\section{Proof of Theorem 1.5}

We now assume that $(x, y, z, m, n)$ is a solution of (1.2) satisfying $2 \mid x, y$ is an odd prime, $m<7, n=5$ and $(x, y, z, m, n) \neq(122,11,3,2,5)$. By Theorem 1.1 , we have $2 \nmid m$. It implies that $m \in\{3,5\}$.

Since $2 \mid x$, we have $2 \nmid z$. Hence, by (3.1) and (3.2), we get (5.1) and

$$
\frac{a^{5}-b^{5}}{a-b}=y^{m} \text {. }
$$

When $m=3$, substitute (5.1) into (7.1), we have

$$
5\left(b^{2}+2 b+2\right)^{2}-4=y^{3} .
$$

But, since $y>1$, by Lemma (2.6), (7.2) is false.

When $m=5$, by (5.1) and (7.1), we have

$$
a^{5}-b^{5}=2 y^{5} .
$$

But, by Lemma 2.11, (7.3) is false. Thus, the theorem is proved.

\section{Acknowledgment}

The fourth author was supported by the Research Fund of Bursa Uludağ University under Project No: F-2020/8. The research of the first and third author was supported in part by grants K115479, K128088 and ANN130909 of the Hungarian National Foundation for Scientific Research and by the projects EFOP-3.6.1-16-2016-00022 and EFOP-3.6.2-16-2017-00015, co-financed by the European Union and the European Social Fund. 


\section{References}

[1] M.A. Bennett, I. Chen, S.R. Dahmen, S. Yazdani, Generalized Fermat equations: a miscellany, Int. J. Number Theory, 11 (2015), 1-28.

[2] M.A. Bennett, P. Minăilescu, S. Siksek, The generalized Fermat equation, Open Problems in Mathematics, (J. F. Nash, Jr. and M. Th. Rassias eds.), Springer, New York (2006), 173-205.

[3] M. A. Bennett and C. M. Skinner, Ternary Diophantine equations via Galois representations and modular forms, Canad. J. Math., 15 (2004), $23-54$.

[4] Y. Bugeaud, On the Diophantine equation $x^{2}-2^{m}= \pm y^{n}$, Proc. Amer. Math. Soc., 125 (1997), 3203-3208.

[5] Y. Bugeaud, On the Diophantine equation $x^{2}-p^{m}= \pm y^{n}$, Acta Arith., 80 (1997), 213-223.

[6] J.H.E. Cohn, The Diophantine equation $x^{n}=D y^{2}+1$, Acta Arith., 106 (2003), 73-83.

[7] H. Darmon and L. Merel, Ternary Diophantine equations via Galois representations and modular forms, J. Reine Angew. Math., 490 (1997), 81-100.

[8] W. Ivorra, Sur les équations $x^{p}+2^{\beta} y^{p}=z^{2}$ et $x^{p}+2^{\beta} y^{p}=2 z^{2}$, Acta Arith., 108 (2003), 327338.

[9] C. Ko, On the Diophantine equation $x^{2}=y^{n}+1, x y \neq 0$, Sci. Sinica 14 (1965), 457-460.

[10] M.-H. LE, Some exponential Diophantine equations I: The equation $D_{1} x^{2}-D_{2} y^{2}=\lambda k^{z}$, J. Number Theory, 55 (1995), 209-221.

[11] P. MihăILESCU, Primary cyclotomic units and a proof of Catalan's conjecture, J. Reine Angew. Math.., 572 (2004), 167-195.

[12] L. J. Mordell, Diophantine equations, London, Academic Press, 1969.

[13] A. Schinzel and R. Tijdeman, On the equation $y^{m}=P(x)$, Acta Arith., 31 (1976), 199-204.

[14] S. Siksek, On the Diophantine equation $x^{2}=y^{p}+2^{k} z^{p}$, Journal de Théorie des Nombres de Bordeaux, 15(2003), 839-846. 
[15] R. TAylor and A. Wiles, Ring-theoretic properties of certain Hecke algebras, Ann. of Math., 141 (1995), 553-572.

[16] A. WiLEs, Modular elliptic curves and Fermat's last theorem, Ann. of Math., 141 (1995), 443-551.

[17] H. Yang AND R. FU, An upper bound for least solution of the exponential Diophantine equation $D_{1} x^{2}-D_{2} y^{2}=\lambda k^{z}$, Int. J. Numb. Theo., 11 (2015), 11071114.

Attila BÉRCZES,

University of Debrecen

Institute of Mathematics

H-4032 Debrecen, Hungary

Email: berczesa@science.unideb.hu

Maohua LE,

Institute of Mathematics, Lingnan Normal College

Zhangjiang, Guangdong, 524048 China

Email: lemaohua2008@163.com

István PINK

University of Debrecen

Institute of Mathematics

H-4032 Debrecen, Hungary

Email: pinki@science.unideb.hu

Gökhan SOYDAN,

Department of Mathematics

Bursa Uludağ University

Görükle Campus, 16059 Bursa, Turkey

Email: gsoydan@uludag.edu.tr 